\title{
Treatment Efficacy and Clinical Effectiveness of EEG Neurofeedback as a Personalized and Multimodal Treatment in ADHD: A Critical Review
}

This article was published in the following Dove Press journal:

Neuropsychiatric Disease and Treatment

\author{
Miguel Garcia Pimenta (D) \\ Trevor Brown (1D ${ }^{2}$ \\ Martijn Arns iD $^{3-5}$ \\ Stefanie Enriquez- \\ Geppert (iD) 1,6 \\ 'Department of Clinical and \\ Developmental Neuropsychology, \\ University of Groningen, Groningen, the \\ Netherlands; ${ }^{2}$ NeuroCare Group, \\ Melbourne, Australia; ${ }^{3}$ Department of \\ Psychiatry, Amsterdam UMC, University \\ of Amsterdam, Location AMC, \\ Amsterdam Neuroscience, Amsterdam, \\ the Netherlands; ${ }^{4}$ Research Institute \\ Brainclinics, Brainclinics Foundation, \\ Nijmegen, the Netherlands; ${ }^{5}$ Department \\ of Cognitive Neuroscience, Faculty of \\ Psychology and Neuroscience, Maastricht \\ University, Maastricht, the Netherlands; \\ ${ }^{6}$ Department of Biomedical Sciences of \\ Cells \& Systems, Section of Cognitive \\ Neuropsychiatry, University of \\ Groningen, Groningen, the Netherlands
}

Correspondence: Miguel Garcia Pimenta Department of Clinical and Developmental Neuropsychology, Faculty of Behavioural and Social Sciences, University of Groningen, Grote Kruisstraat 2/I, Groningen, 97/2 TS, the Netherlands

Tel +3I 503636537

Emailm.garcia.pimenta@rug.nl
Purpose: Recent reviews have proposed that scientifically validated standard EEG neurofeedback (NF) protocols are an efficacious and specific treatment for attention-deficit hyperactivity disorder (ADHD). Here, we review the current evidence for the treatment efficacy and clinical effectiveness of NF in ADHD to investigate whether NF treatment personalization (standard protocols matched to the electrophysiological features of ADHD) and combination with other interventions (psychosocial, sleep hygiene and nutritional advice) might yield superior long-term treatment outcomes relative to non-personalized NF and medication monotreatments.

Methods: The electronic databases PubMed and PsycINFO were systematically searched using our key terms. Of the 38 resulting studies, 11 randomized controlled trials (RCTs) and open-label studies were eligible for inclusion. Studies were analyzed for effect sizes and remission rates at the end of treatment and at follow-up. The effects of personalized and multimodal NF treatments were compared to non-personalized NF monotreatments and with two benchmark medication studies.

Results: The analysis of RCTs indicated that the long-term effects of personalized NF interventions were superior to non-personalized NF and comparable to those of medication alone or in combination with behavioral intervention. The analysis of open-label trials further indicates that the interaction of NF with parental interventions, sleep and nutritional advice might yield superior clinical effectiveness relative to NF and medication monotreatments.

Conclusion: Personalized and multimodal NF interventions seem to yield superior treatment efficacy relative to NF alone and superior clinical effectiveness relative to medication. We propose that treatment outcomes may be further enhanced by adjusting NF non-specific factors (eg, reinforcement contingencies) to specific ADHD characteristics (eg, reward sensitivity). Future NF research should focus on the systematic evaluation of the treatment outcomes of personalized and multimodal treatments.

Keywords: neurofeedback, ADHD, treatment efficacy, effectiveness, personalized, multimodal

\section{Introduction}

Attention-deficit hyperactivity disorder (ADHD) is a highly prevalent and impairing neurodevelopmental disorder ${ }^{1}$ characterized by persistent inattention, impulsivity and hyperactivity in multiple domains of functioning. From a neurocognitive point of view, ADHD has been associated with deficits in cognitive control, delay 
aversion, reward sensitivity and timing. ${ }^{2-4}$ Its diverse clinical presentation and neuropsychological profile suggests an heterogenous and multifactorial causation ${ }^{5}$ including genetic and environmental factors. Besides the lifelong implications for mental health, ${ }^{6}$ ADHD can represent a significant psychological and economic burden for families and society (eg, in terms of medical treatments and lost productivity) justifying the consideration of longterm cost-effective interventions. ${ }^{7-9}$ Although psychopharmacological treatments have been shown to be most effective $^{10}$ and relatively affordable compared to psychosocial interventions, the benefits seem not to persist in the long-term. ${ }^{11,12}$ Such results have encouraged research to focus on long-term effectiveness of multimodal treatments ${ }^{11,13,14}$ and their inclusion in treatment guidelines. ${ }^{15}$ In this context, neurofeedback (NF) has been considered a promising non-pharmacological intervention as a stand-alone ${ }^{16-18}$ or in combination with other treatment modalities. ${ }^{19,20}$

$\mathrm{NF}$ is a neuromodulation technique based on a braincomputer interface (BCI) by which pre-selected parameters of brain activity (eg, electroencephalographic (EEG) frequencies) are directly relayed back to the individual in real-time via goal-directed visual and auditory cues. Through NF, brain activity underlying cognition and behaviour is targeted based on mechanisms of reinforcement and procedural skills learning. NF can be tailored to individual differences in the brain signal of interest and flexibly integrated with other psychosocial and behavioral treatments. ${ }^{21} \mathrm{NF}$ effects are probably the result of complex multifactorial mechanisms including both specific and non-specific factors and their interaction. ${ }^{22}$ This may include non-specific factors such as (1) expectations regarding the technique (eg, technological acceptance, placebo effect), (2) secondary reinforcement associated with the target brain activity (eg, token economy system), (3) and primary reinforcement of behaviors adequate to the context of NF but not related to the brain signal (eg, learning to sit still and to prevent electromuscular artifacts). ${ }^{23-25}$ The clinical implementation of NF also involves general non-specific factors common to other psychosocial interventions (eg, praise and supportive role of the therapist) which are not only facilitative of the learning process via primary reinforcement of adequate behaviors but also rewarding in itself. ${ }^{26}$ Importantly, these factors are likely present in multiple interventions and their interaction with ADHD individual differences needs to be considered when evaluating the effects of personalized and multimodal NF interventions. The current review aims to evaluate the treatment efficacy and clinical effectiveness of personalized and multimodal treatments including NF, while considering their flexibility in addressing the heterogeneous and multifactorial nature of ADHD.

\section{Criteria of Treatment Efficacy and Clinical Effectiveness}

According to the American Psychological Association (APA) psychological treatments are rated by their (1) "treatment efficacy" concerning the scientific evidence and (2) "effectiveness" referring to the applicability or clinical utility. ${ }^{27}$ If the intervention is deemed the best treatment alternative in economic terms, then the treatment is cost-effective. Following Southam-Gerow and Prinstein ${ }^{28}$ and Tolin et $\mathrm{al}^{29}$ suggested updates, Arns et $\mathrm{al}^{30}$ recently proposed a stricter version of the original APA guidelines that explicitly considers both the long-term effectiveness and the remission rates in the evaluation of the clinical significance of evidence-based treatments.

\section{Methods}

\section{Study Selection}

Figure S-1 (Supplementary material) presents the Preferred Reporting Items for Systematic Reviews and Meta-Analyses (PRISMA) flow diagram of study selection in the current review. The literature was searched for studies that examined the efficacy and effectiveness of EEG NF in ADHD using the electronic databases PubMed and PsycINFO with the following Boolean logic terms: ("ADHD" or "attention deficit hyperactivity disorder" or "attention deficit-hyperactivity disorder") AND ("neurofeedback" or "eeg biofeedback") AND ("trial" or "study" or "randomized controlled trial" or "RCT") AND ("efficacy" or "effectiveness") AND ("rating scale" or "scale" or "parent rating" or "teacher rating") limited to the last five years (2016-2020). Systematic reviews and meta-analyses resulting from this search were examined to identify potentially missed publications. The search through the databases identified 32 abstracts to which six other sources were added based on the most recent systematic review. ${ }^{30}$ Duplicates were excluded, abstracts were screened and the full text examined by the first and second authors based on the following inclusion criteria: 1) multicentre randomized controlled trials (RCTs) published in peer-reviewed journals available in English language; 2) 
primary diagnosis of ADHD; 3 ) mean child age $<18$ years old; and 4) NF treatment used one of the well-investigated and specific standard protocols - Theta/beta ratio (TBR), Sensorimotor Rhythm (SMR), and/or Slow Cortical Potentials (SCP) - as proposed by Arns, Heinrich and Strehl. ${ }^{31}$ The screening of the 38 records resulted in the exclusion of nine articles: five for being systematic reviews or meta-analyses; two because the primary diagnosis was not ADHD; one because the participants had a mean age higher than 18 years old, and one because it was not available in the English language. During the screening of the full text of the remaining 27 records, several articles belonging to the same group of authors were identified and clustered. Then, similarities in sample and outcome measures were examined resulting in the exclusion of one article in which primary parental and teacher's ratings were repeated in other sources of the same cluster. Finally, the full text of the remaining articles was examined resulting in the exclusion of 15 other articles which did not meet criteria for being multicentre RCTs and/or not using standard NF protocols. Table S-1 (Supplementary material) presents a list of excluded articles with reasons. Eleven articles were selected for the current critical review.

In order to provide a frame of reference for the efficacy and effectiveness of personalized and multimodal NF interventions, identical outcome measures were extracted from two multicenter studies examining traditional treatments of ADHD in children. The benchmark multicenter NIMH Multimodal Treatment Study of Children with ADHD (MTA) ${ }^{32}$ was chosen as a reference RCT to determine treatment efficacy. Clinical effectiveness was operationalized as the generalizability of NF efficacy to everyday clinical practice in open-label studies and assessed in relation to the nonindustry sponsored multi-center open-label International Study to Predict Optimized Treatment in ADHD (iSPOT-A). ${ }^{33,34}$

\section{Outcome Measures}

In line with Arns et $\mathrm{al}^{30}$ the current review followed the recent recommendations of Tolin et $\mathrm{al}^{29}$ in which three sets of outcome measures were adopted to assess treatment efficacy and effectiveness of NF: The first outcome measure was the acute pre-post treatment (pre-post) effect size (ES), expressed as Cohen's d, which reflects the improvement of the group from pre-treatment to post treatment on the primary endpoint in a given study.
Conventionally, an ES $>0.3$ is considered a small clinical effect, an ES $>0.5$ considered a medium sized effect and an ES $>0.8$ considered a large clinical effect. Moreover, long-term pre-treatment to follow-up (pre-FU) ES was considered. Finally, remission rate (ie, loss of diagnostic status) was considered. This was defined as an ADHD rating scale item mean of $\leq 1.0,{ }^{35,36}$ published for the multi-center NIMH Multimodal Treatment Study of Children with ADHD (MTA). ${ }^{35}$ When remission was not published, the authors were contacted (up to two times) to request remission rates. These criteria were fulfilled by the pooled quantitative EEG (QEEG) informed open-label studies by Arns et al and Krepel et $\mathrm{al}^{37,38}$ the combined SCP and TBR RCT by Gevensleben et $\mathrm{al}^{39,40}$ the SCP RCT by Strehl et $\mathrm{al}^{41}$ and the SCP RCT by Aggensteiner et $\mathrm{al}^{42}$ (reporting the pre-FU results of Strehl et al). However, for various reasons the requests could not be fulfilled by the studies of Kropotov et $\mathrm{al}^{43}$ Monastra et $\mathrm{al}^{20}$ Steiner et $\mathrm{al}^{44}$ and no response was obtained from Geladé et al. ${ }^{45}$ For comparison, the same information about treatment outcomes was obtained for the two benchmark medication studies. ${ }^{33,35}$ For an overview of the studies' characteristics included in the present review see Table 1 .

\section{Results}

\section{Treatment Efficacy and Clinical Effectiveness of Pharmacological Treatment in ADHD}

The treatment outcomes for all studies considered in the current review are presented in Figure 1. In the NIMHMTA trial, the combined treatment shows the highest clinical significance with $68 \%$ remission rate and the largest pre-post ES of 1.63 which declined on FU (ES of about 1.4; no information about remission rates). Medication as a stand-alone treatment shows good clinical and statistical significance $(56 \%$ remission rate, prepost and pre-FU treatment ES around 1.4). Remission rates, and statistical significance are lower in the multicomponent behavior therapy (remission rate: $34 \%$, with pre-post and pre-FU treatment ES of about 1) and in the community care (remission rate: $25 \%$, with pre-post and pre-FU treatment ES of about 0.9-1). Despite the strong pre-post ES (1.09) the iSPOT-A shows that methylphenidate had a lower clinical effectiveness than that obtained during RCT conditions as shown by the lower 


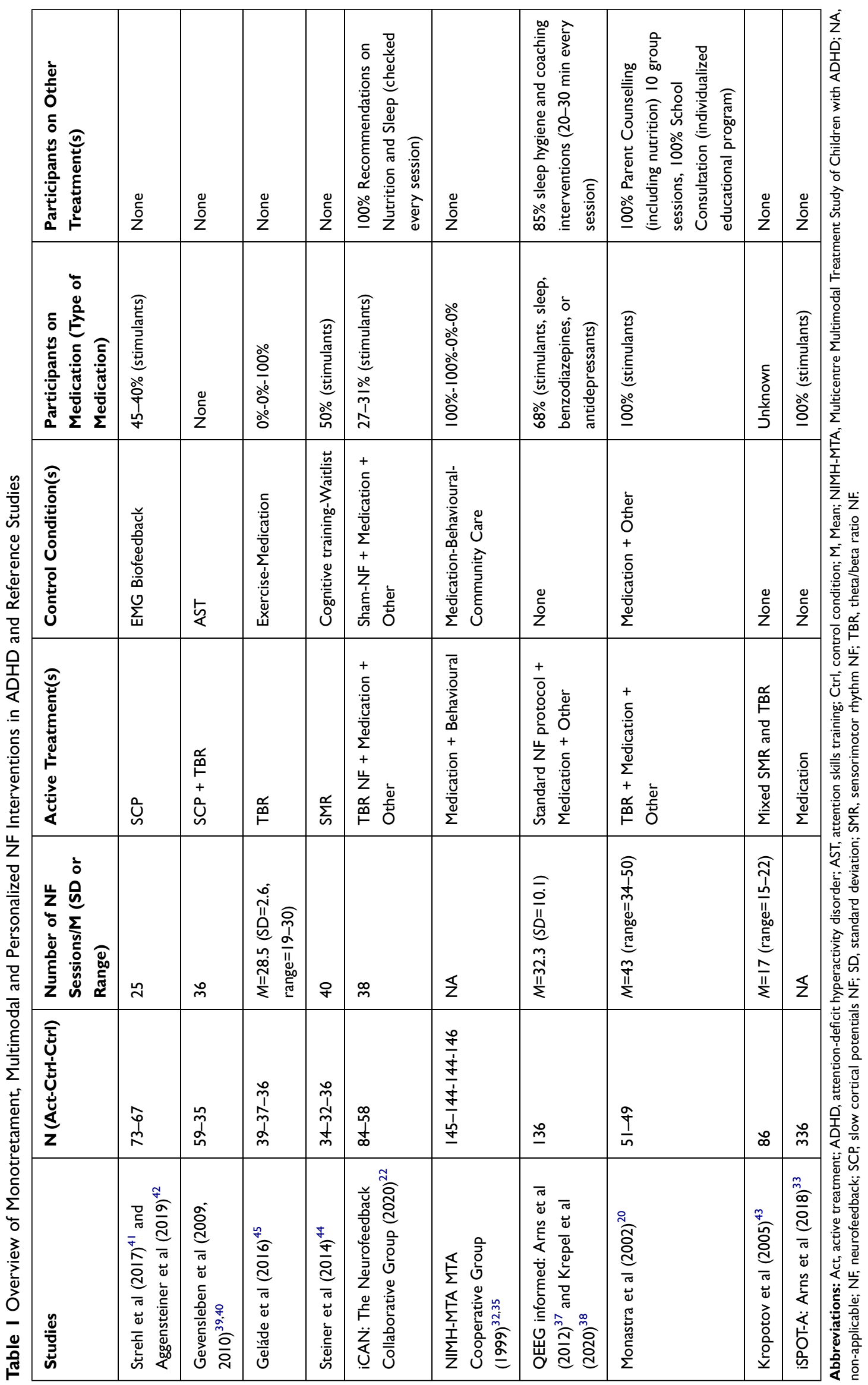




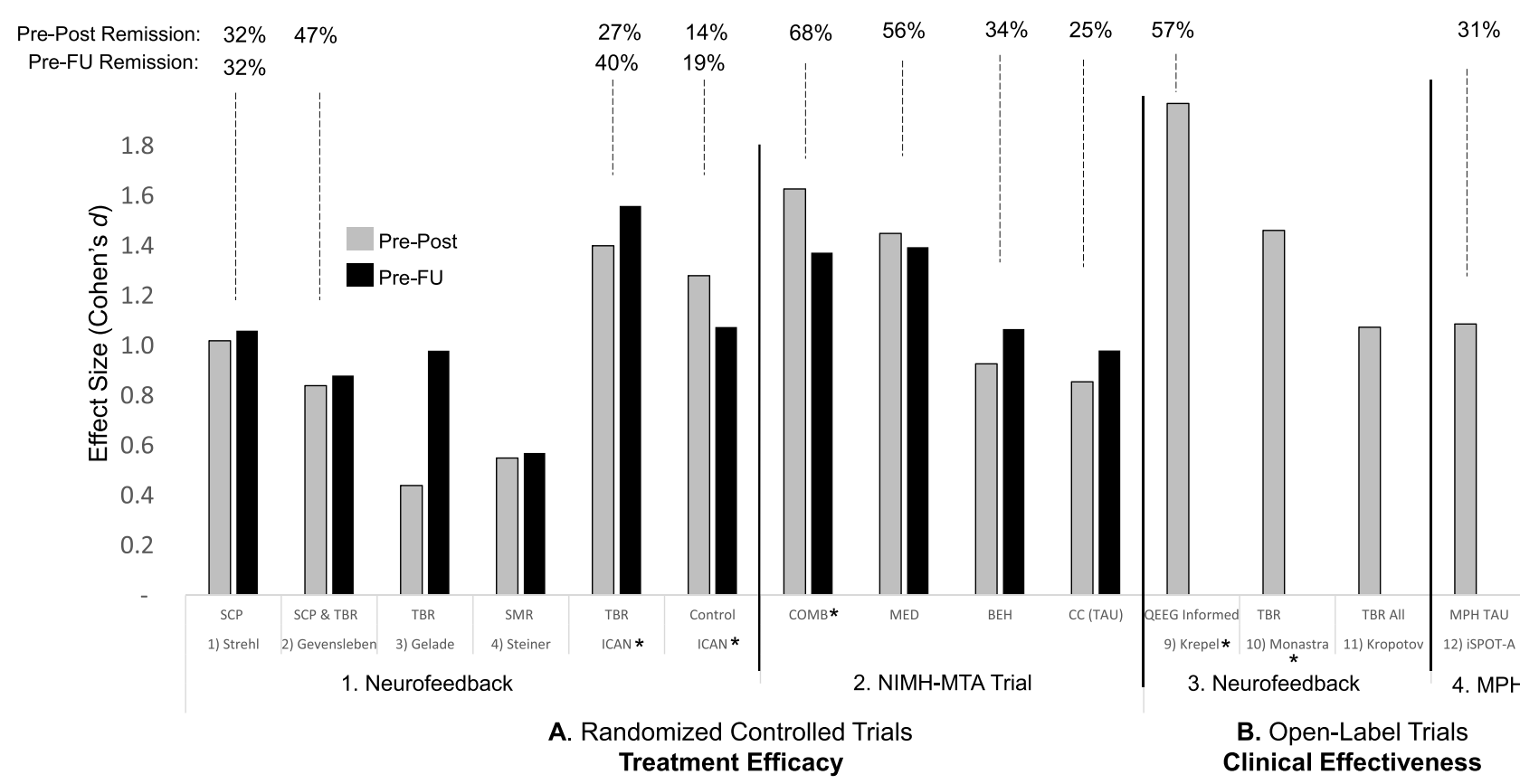

Figure I Comparative treatment efficacy and effectiveness of treatments in ADHD. Pre-post treatment ES (Cohen's $d$ ) for parent rated overall ADHD symptom improvement (grey, Pre-Post) and from pre-treatment to follow-up (black, Pre-FU); pre-post and pre-FU treatment remission rates are listed above (top line). On the left the results for Treatment Efficacy (A. Randomized Controlled Trials) are depicted for I. Neurofeedback RCT's based on standard protocols Slow Cortical Potentials (SCP), Theta/beta ratio (TBR), Sensorimotor Rhythm (SMR) and for 2. NIMH-MTA Trial treatment arms (Combined treatment (COMB), Medication only (MED), Multicomponent Behaviour Therapy (BEH) and Community Care (CC). On the right the results for Clinical Effectiveness (B. Open-Label Trials) are depicted for 3. Neurofeedback open-label trials using treatment personalization based on QEEG or TBR and for 4. Methylphenidate (MPH) treatment as usual (TAU) open-label data from the iSPOT-A study. The multimodal NIMH-MTA and the personalized and multimodal NF studies are marked with *.

remission rates after treatment $(31 \%$ in the iSPOT-A against $56 \%$ in the MTA).

\section{Treatment Efficacy and Clinical Effectiveness of Neurofeedback}

As shown in Figure 1, seven RCTs focusing on standard protocols met inclusion criteria for the current review. First, Strehl et $\mathrm{al}^{41}$ utilized the SCP protocol and compared it to EMG biofeedback as an active control group. They demonstrated a large ES of 1.02 for acute pre-post treatment and 1.06 for long-term pre-FU effects, apart from a remission rate of $32 \%$ pre-post which remained stable after pre-FU as reported by Aggensteiner et al. ${ }^{42}$ Second, Gevensleben et al ${ }^{39,40}$ compared SCP and TBR to cognitive training, demonstrating large ES for prepost (0.84) and for pre-FU (0.88), as well as a $47 \%$ remission rate. Unfortunately, remission rates were not available for pre-FU. Third, Geladé et $\mathrm{al}^{45}$ utilized TBR and compared this to exercise and methylphenidate, with medium to large EF for pre-post effects (0.44) and preFU (0.98). Fourth, Steiner et $\mathrm{al}^{44}$ assessed an SMR protocol implemented in a school setting in comparison to cognitive training and a waitlist, showing medium
ESs for pre-post effects (0.55), and for pre-FU (0.57). Fifth, in the recently published International Collaborative ADHD Neurofeedback (iCAN) study, ${ }^{22}$ TBR was compared to sham-NF, demonstrating large ES for pre-post (1.4) and pre-FU (1.56) with a remission rate of $27 \%$ pre-post which increased to $40 \%$ at follow up. However, the sham-NF group also had large ES for pre-post (1.28), and pre-FU effects (1.08), despite reporting smaller remission rates of $14 \%$ at pre-post and $17 \%$ pre-FU.

Regarding clinical effectiveness, four open-label studies were included; however, no follow-up information is available for any of those studies. First, Monastra et $\mathrm{al}^{20}$ used TBR NF which resulted in a high ES for pre-post effects (1.46). Second, Kropotov et $\mathrm{al}^{43}$ used a relative beta protocol, and similarly showed high ES for pre-post effects (1.07). Third, Krepel et $\mathrm{al}^{38}$ used individual QEEG to assign participants to one of the standard protocols thus replicating Arns et $\mathrm{al}^{37}$ in a multicentric study with a large sample. When the participants of these two open-label studies were pooled together, the acute prepost ES was large (1.97) and a remission rate of 57\% was observed. 


\section{Discussion}

\section{Long-Term Efficacy and Effectiveness of Pharmacological and NF Interventions}

Taken together our results suggest that NF RCTs show lower clinical significance (with remission rates between $14 \%-47 \%$ and ES between 0.44-1.56) compared to the NIMH-MTA Trial (with remission rates between $25 \%$ $68 \%$ and ES between 0.63-0.86). Interestingly, in the case of NF, ESs consistently increased (or were maintained) from pre-post to pre-FU treatment, while for the MTA medication arms (either in isolation or combined with other treatments), decreases in treatment effects were observed. Based on the available evidence, the ESs remained stable or increased over the long term thus indicating a strengthening of effects for NF over time, without any additional treatment. The NF open-label trials also demonstrate that the benefit obtained in clinical settings is overall similar or better relative to NF clinical trials ('effectiveness' based on standard protocols), whereas the clinical effects of the open-label iSPOT-A tend to be lower relative to the medication arm of the RCT MTA Trial. In the following sections, we advance the novel proposal that the incremental benefits of NF in the long term and in the clinical settings described above may be explained by its potential for treatment personalization and for synergistic interaction with other treatment modalities.

\section{Increasing the Effects of Neurofeedback Through Personalization \\ NF Personalization to EEG Features Increases Treatment Efficacy and Effectiveness}

The personalization of NF parameters to individual differences may account for the superior effectiveness relative to randomization of participants to one-size-fits-all standard NF protocols. Current evidence suggests that individually adjusting the NF treatment protocol to the patient baseline QEEG signal characteristics was associated with superior treatment outcomes relative to random assignment of participants to standard NF protocols and comparable remission rates to medication. The results of three NF personalized studies are shown in Figure 1. In Monastra et $\mathrm{al}^{20}$ and $\mathrm{iCAN}^{22}$ studies ADHD children were pre-selected based on elevated theta-beta ratios and subsequently treated with TBR NF. Similarly, in the two open-label studies by Arns et al and Krepel et $\mathrm{al}^{37,38}$ the baseline QEEG informed the decision to assign the ADHD patients to one of the three standard protocols (SCP, SMR or TBR). As shown in Figure 1, the pre-post ESs were larger in the NF personalized interventions (1.40 for the TBR iCAN, 1.46 for the TBR and 1.97 for the QEEG informed) than when participants were randomized to non-personalized standard protocols (1.02 for SCP, 0.84 for SCP \& TBR, 0.55 for TBR and 0.44 for SMR). Moreover, the pooled pre-post remission rate of $57 \%$ of the two open-label studies ${ }^{37,38}$ was comparable to the $56 \%$ obtained in the reference NIMH-MTA Medication Management treatment and superior to the $31 \%$ observed in the open-label iSPOT-A. ${ }^{33,34}$ That remission rate was also significantly higher than that observed in the iCAN study (27\%) which examined only the effects of personalized TBR but not of other standard NF protocols. Taken together these studies suggest that a stratification approach based on the EEG likely enhances clinical response to standard NF protocols. Essentially, this can be seen as a way to enhance the signal-to-noise ratio for NF, thus increasing the trainability of the signal. This is in line with current evidence of interindividual differences in the generation and successful control of intrinsic patterns of neural activity. ${ }^{46-48}$ Future RCTs should systematically assess the effects of standard NF protocols (TBR, SCP and SMR) relative to personalization (personalized vs nonpersonalized) in a large sample. A personalized approach would recommend, for example, that a well investigated protocol other than TBR NF (eg, SMR or SCP) be selected when patients do not present TBR elevations in the QEEG.

NF Personalization to ADHD Features May Further Increase Treatment Efficacy and Effectiveness

As described in previous sections, non-specific factors may account for a significant proportion of NF treatment effects as demonstrated by the large pre-post ES of the iCAN control group. ${ }^{22}$ Here we propose that the efficacy of NF in ADHD may be further increased by matching non-specific treatment factors to individual differences among the multifactorial and heterogenous ADHD diagnosis. ADHD children show higher reward sensitivity than typically developing controls as demonstrated by (1) improved instrumental learning with shorter time intervals between the response and the reinforcer, ${ }^{49}$ (2) preference for immediate rather than large, delayed rewards ${ }^{50}$ and (3) increased feedback monitoring and motivation when feedback is coupled with reward. ${ }^{51}$ Higher reward sensitivity in ADHD children has been associated with increased motivation, sustained attention and arousal in response to reinforcement especially during repetitive and non-novel 
tasks ${ }^{2,4,52-55}$ with similar characteristics to NF. These reinforcement contingencies can be found in several RCT studies that used psychosocial and behavioral treatments $^{40-42,44}$ and sham-NF as control conditions. ${ }^{56}$ As in bona fide-NF, sham-NF also provide primary and secondary reinforcement of NF non-specific behaviors (eg, motor inhibition and monetary reward respectively) as well as an equivalent amount of general non-specific reinforcement (eg, praise by the therapist). In face of the above, future studies are needed to determine the extent to which individual differences in reward sensitivity among ADHD children may moderate NF treatment outcomes in both bona fide and control conditions.

Another non-specific NF treatment effect that may interact with specific ADHD features is the external regulation and reinforcement of appropriate motor activity. The instructions to reduce motor activity in both bona fide and sham-NF conditions, necessary for the acquisition of a clean electroencephalographic signal, also create opportunities for behavioral regulation. By receiving feedback on electromyographic (EMG) activity, verbal instructions, coaching and reward contingent on appropriate motor behavior (ie, keeping jaw and face muscles relaxed to avoid artifacts, sitting still, maintaining an upright posture, and attending to the screen), NF constitutes an approximation to surface EMG biofeedback which has been shown to be associated with small but significant effects in the regulation of behavior and attention in ADHD children. ${ }^{41,57-59}$ For example, EMG biofeedback had similar positive effects to NF on symptoms of hyperactivity as rated by parents ${ }^{41}$ and on neuropsychological measures of attention (eg, RT mean and RT variability). ${ }^{59}$ Taken together this evidence suggests that the EMG biofeedback component of NF might have a small yet significant role in the regulation of motor behavior and, therefore, in decreasing related ADHD symptoms. Future research is needed to clarify whether the non-specific EMG component of NF may have a differential positive impact on specific ADHD behaviors (eg, hyperactivity) and whether this can be predicted from individual differences in ADHD subtype.

\section{Neurofeedback in the Context of Multimodal Interventions}

In recent years, the wider range of available treatment modalities for ADHD provided new possibilities for customizing interventions to patient characteristics and needs. As shown in previous sections, multimodal interventions (eg, MTA Trial) combining medication and behavioral treatments were associated with better treatment efficacy relative to monotherapy, suggesting additive and/or synergistic effects. However, as shown in Figure 1, the long-term effects indicate that the MTA combined treatment brings no advantage relative to medication alone and is less cost-effective. In face of this evidence, it has been proposed that clinical decision in favor of a multimodal intervention should be informed by patient characteristics. ${ }^{60}$ However, no studies to date have provided empirical support to these recommendations. In the remainder of this section, we will examine the current evidence regarding the clinical efficacy of multimodal interventions including NF treatments focusing on its additive and synergistic effects with evidencebased pharmacological and behavioral treatments and other promising adjunctive treatments such as school interventions nutrition and sleep advice. Basic information on relevant studies characteristics can be found in Table 1.

\section{Multimodal Neurofeedback Relative to Neurofeedback Monotreatment}

The combination of NF with other treatment modalities seems to be associated with superior effects relative to NF alone. As shown in Figure 1 (1. Neurofeedback), the multimodal iCAN study including TBR in combination with counseling on sleep and nutrition showed considerably higher pre-post and pre-FU treatment ESs relative to all other NF monotreatments. The multimodal TBR outperformed the best performing SCP monotreatment, when both pre-post (1.40 against 1.02) and pre-FU treatment (1.56 against 1.06) ES were considered. These results are, however, difficult to interpret because of the differences in NF protocols, the smaller number of sessions in SCP monotreatment relative to the multimodal TBR and varying percentage of ADHD children on medication during NF treatment (see Table 1). When the same NF protocol is considered (ie, TBR), the effects of the multimodal treatment clearly exceed those of the monotreatment ${ }^{45}$ (pre-post: 1.40 against 0.44 ; pre-FU: 1.56 against 0.98 ). However, the lower number of NF sessions in Geladé et $\mathrm{al}^{45}$ study cannot be ruled out as a possible explanation for the apparent superiority of the multimodal treatment. When studies with a similar number of NF sessions are used as a comparison (ie, Gevensleben et $\mathrm{al}^{40}$ and Steiner et $\mathrm{al}^{44}$ ), the TBR multimodal treatment still shows higher 
pre-to post treatment ESs suggesting that the number of NF sessions per se does not explain the superior treatment efficacy.

When open-label studies are considered (see Figure 1, 3. Neurofeedback), multimodal interventions such as those reported by Krepel et $\mathrm{al}^{38}$ (based on more than one standard NF protocol, sleep hygiene and coaching) and by Monastra et $\mathrm{al}^{20}$ (based on TBR, parental counseling and school interventions) seem to yield superior results relative to the NF monotreatment (mixed SMR and relative beta uptraining) reported by Kropotov et al. ${ }^{43}$ However, the significantly lower number of NF training sessions in the latter study needs to be considered as a possible explanation for the smaller pre-post-treatment ES (see Table 1 for number of NF sessions in each study). Taken together, the results from both RCT and open-label studies seem to suggest that NF as part of a multimodal treatment is associated with better treatment outcomes relative to NF monotreatment. However, it is unclear whether this superiority might be related to the higher number of sessions or the personalization of the NF treatment which coincidentally was only implemented in the multimodal treatments.

\section{Multimodal Neurofeedback: Possible Interactions with Parental, Sleep and Nutrition Interventions}

Current evidence suggests that NF might carry additional treatment benefits when associated with more comprehensive treatment plans combining medication with psychosocial treatments. Monastra et $\mathrm{al}^{20}$ suggested that around 40 sessions of TBR NF has an additional positive shortterm impact on core ADHD symptoms and might contribute to reduce the need for medication when combined with other interventions. However, because Monastra et al's study did not include a control condition it remains unclear whether benefits of the additional intervention were related to the NF specific or non-specific effects. This study provided evidence that at least part of the NF additive gains could be related to the interaction between NF and parental training. Among the children that received NF in addition to other interventions, only those whose parents adopted a systematic parenting style (ie, consistent use of reinforcement and response cost) showed improvements in inattention and hyperactivity symptoms when assessed both under medication and after a one-week washout from medication. However, these positive effects of parenting style were not evident on the teacher ratings of inattention and hyperactivity suggesting that the effects are context specific or, alternatively, biased by parental expectations. Furthermore, it remains unclear which aspects of parental intervention might have contributed to potentiate and consolidate the effects of NF. The benefits of this interaction might be related to the modification of parenting styles (eg, through the systematic use of positive reinforcement), to the higher parental involvement and motivation for treatment (and consequent stronger placebo effect) or to the generalization of specific behavioral regulation strategies to the home environment.

Finally, the combination of NF with counseling on sleep, ${ }^{38}$ nutrition, ${ }^{20}$ or both $^{22}$ may have contributed to the overall treatment effects of several NF multimodal RCT and open-label studies. In fact, both Monastra et $\mathrm{al}^{20}$ and the iCAN study ${ }^{22}$ stressed the importance of- and made specific recommendations regarding breakfast in line with current evidence that poor nutrition is an environmental risk factor for ADHD. ${ }^{61}$ Although the additional benefits of the nutritional recommendations could not be tested independently from the effects of other interventions there is evidence to suggest that nutritional interventions per se can have a small yet significant positive effect on ADHD symptoms. ${ }^{16}$ Furthermore, recent multimodal interventions have combined NF and counselling to improve the quantity and quality of sleep in line with the increasing evidence of a strong association between ADHD and sleep disorders, ${ }^{62}$ the effects of NF in the regulation of sleep spindle circuitry and circadian networks in $\mathrm{ADHD},{ }^{63}$ and the finding that ADHD symptom reduction following NF may be mediated by improvements in sleep. ${ }^{64}$

In conclusion, the large treatment effects observed in both bona fide and sham-NF interventions can be the result of adding up the small effects of several treatment modalities and their specific and non-specific components. Moreover, the superior effectiveness of multimodal interventions including NF, parental interventions, sleep and nutritional advice in comparison to NF monotreatment suggest that these interventions might have contributed additively or synergistically to the overall treatment effects. On the other hand, multimodal approaches may increase the probability of addressing multiple factors contributing to the causation or maintenance of the ADHD symptoms. Further studies are needed to systematically investigate the hypothesis that NF and other treatment modalities may work synergistically to improve the treatment outcomes. In order to optimize treatment selection, future studies should also attempt to clarify which ADHD characteristics, associated functional difficulties 
and patterns of comorbidities are more likely to benefit from combining NF with parental interventions as well as with sleep and dietary advice. Additionally, future research should attempt to determine whether multiple interventions are optimally delivered simultaneously and/or sequentially as recent evidence suggests that the sequencing of medication and behavioral interventions may be crucial for treatment response and prevention of medication side-effects. ${ }^{65}$

Apart from clinical effectiveness, treatment selection needs also to consider cost-effectiveness. However, the costeffectiveness of NF relative to medication and behavioral therapy is not yet clearly established. Since ADHD is a neurodevelopmental disorder that often persists into adulthood $^{3}$ the long-term effects of treatment are of paramount importance in determining the cost-effectiveness. In the United States, the total economic burden over the course of child development (excluding treatment costs) has been estimated to be $\$ 15,036$ per child with ADHD vs $\$ 2848$ per child without ADHD. ${ }^{7}$ In Germany, for example, the yearly medical cost involving inpatient care, psychotherapy and psychiatric consultations of patients with ADHD amount to $€ 1500$ and to $€ 2800$ when comorbidities were present. ${ }^{8}$ According to estimations performed by Arns et al, personalized and multimodal NF consisting of 30-40 sessions would cost in total between US $\$ 4000$ and $\$ 6000$ (based on hourly fees in developed economies), while the costs of medication excluding inpatient care, pediatric or psychiatric consultation would amount to $\$ 3500$ to $\$ 7000$ for periods ranging between 5 and 10 years (based on an average cost of $\$ 2$ per day). ${ }^{30}$ Although systematic research is needed regarding long-term effects, preliminary evidence suggests that personalized and multimodal NF interventions (ie, the QEEG informed study by Krepel et al and the study by Monastra et al) were associated with higher clinical effectiveness relative to medication monotreatment (ie, the iSPOT-A study) with comparable treatment costs. Taking into account its short treatment duration and long-term clinical effectiveness, NF may be more cost-effective than traditional medical approaches when all costs along the developmental trajectory are considered. ${ }^{30}$

\section{Conclusions}

The current study expands and reinforces the previous conclusions that NF standard protocols can be considered as well-established and "efficacious and specific" with medium to large ES and $32 \%$ to $47 \%$ remission rates, as well as sustained effects on FU. Moreover, our analysis indicates that the remission rates of personalized NF treatment with standard NF (57\%) are superior to methylphenidate in an open-label trial (31\%) and equivalent to monotreatment with medication in a controlled research setting (56\%). The superior effects of the iCAN RCT compared to the remaining NF RCTs, as well as the Krepel et $\mathrm{al}^{38}$ open-label study to the remaining NF studies, provides evidence for the advantage of the personalization of standard protocols to the specific EEG features of the participants, with clear implications for clinical practice. The comparison between multimodal NF interventions to $\mathrm{NF}$ monotreatments is also supportive of additive or synergistic effects of combining NF with other interventions, namely parental, sleep and nutrition interventions. Non-specific treatment factors, such as reinforcement contingencies and the training of motor behavior during NF provide further opportunities for the personalization of NF training to match the heterogeneous presentation of ADHD and calls for systematic future research.

\section{Author Contributions}

All authors made a significant contribution to the work reported, whether that is in the conception, study design, execution, data interpretation, or in all these areas; took part in drafting, revising or critically reviewing the article; gave final approval of the version to be published; have agreed on the journal to which the article has been submitted; and agree to be accountable for all aspects of the work. MA acquired and analyzed the data.

\section{Disclosure}

Miguel Garcia Pimenta is a guest lecturer in neurofeedback courses for neuroCare Group (Munich, Germany). Trevor Brown is employed by neuroCare Group (Melbourne, Australia) who offer neurofeedback treatment within their clinics.

Martijn Arns is an unpaid chairman of the Brainclinics Foundation, a minority shareholder in and adviser to neuroCare Group (Munich, Germany), and a co-inventor on 4 patent applications related to EEG, neuromodulation and psychophysiology, but receives no royalties related to these patents; Research Institute Brainclinics received research funding from Brain Resource (Sydney, Australia), Urgotech (Paris, France) and neuroCare Group (Munich, Germany), and equipment support from Deymed, neuroConn, Brainsway and Magventure. Stefanie Enriquez-Geppert declares no competing interests. 
The authors report no other conflicts of interest related to this work.

\section{References}

1. Polanczyk G, De Lima MS, Horta BL, Biederman J, Rohde LA. The worldwide prevalence of ADHD: a systematic review and metaregression analysis. Am $J$ Psychiatry. 2007;164(6):942-948. doi:10.1176/ajp.2007.164.6.942

2. Luman M, Oosterlaan J, Sergeant JA. The impact of reinforcement contingencies on AD/HD: a review and theoretical appraisal. Clin Psychol Rev. 2005;25(2):183-213. doi:10.1016/j.cpr.2004.11.001

3. Mostert JC, Onnink AMH, Klein M, et al. Cognitive heterogeneity in adult attention deficit/hyperactivity disorder: a systematic analysis of neuropsychological measurements. Eur Neuropsychopharmacol. 2015;25(11):2062-2074. doi:10.1016/j.euroneuro.2015.08.010

4. van Hulst BM, de Zeeuw P, Durston S. Distinct neuropsychological profiles within ADHD: a latent class analysis of cognitive control, reward sensitivity and timing. Psychol Med. 2015;45(4):735-745. doi:10.1017/S0033291714001792

5. Faraone SV, Biederman J. Neurobiology of Attention Deficit/ Hyperactivity Disorder. In: Charney DS, Buxbaum JD, Nestler EJ, editors. Neurobiology of Mental Illness. 4th ed. Oxford University Press; 2013:1034-1047.

6. Hechtman L, Swanson JM, Sibley MH, et al. Functional adult outcomes 16 years after childhood diagnosis of attention-deficit/hyperactivity disorder: MTA results. $J$ Am Acad Child Adolesc Psychiatry. 2016;55(11):945-952.e2. doi:10.1016/j.jaac.2016.07.774

7. Zhao X, Page TF, Altszuler AR, et al. Family burden of raising a child with ADHD. $J$ Abnorm Child Psychol. 2019;47 (8):1327-1338. doi:10.1007/s10802-019-00518-5

8. Libutzki B, Ludwig S, May M, Jacobsen RH, Reif A, Hartman CA. Direct medical costs of ADHD and its comorbid conditions on basis of a claims data analysis. Eur Psychiatry. 2019;58:38-44. doi:10.1016/j.eurpsy.2019.01.019

9. Hakkaart-van Roijen L, Zwirs BWC, Bouwmans C, et al. Societal costs and quality of life of children suffering from attention deficient hyperactivity disorder (ADHD). Eur Child Adolesc Psychiatry. 2007;16(5):316-326. doi:10.1007/s00787-007-0603-6

10. Cortese S, Adamo N, Del Giovane C, et al. Comparative efficacy and tolerability of medications for attention-deficit hyperactivity disorder in children, adolescents, and adults: a systematic review and network meta-analysis. Lancet Psychiatry. 2018;5(9):727-738. doi:10.1016/ S2215-0366(18)30269-4

11. Molina BS, Hinshaw SP, Swanson JM, et al. The MTA at 8 years: prospective follow-up of children treated for combined type ADHD in a multisite study. J Am Acad Child Adolesc Psychiatry. 2009;48 (5):484-500. doi:10.1097/CHI.0b013e31819c23d0

12. Jensen PS, Arnold LE, Swanson JM, Vitiello B, Abikoff HB. 3-year follow-up of the NIMH MTA study. $J$ Am Acad Child Adolesc Psychiatry. 2007;46(8):989-1002. doi:10.1097/ CHI.0b013e3180686d48

13. Lam AP, Matthies S, Graf E, et al. Long-term effects of multimodal treatment on adult attention-deficit/hyperactivity disorder symptoms: follow-up analysis of the COMPAS trial. JAMA Netw open. 2019;2 (5):e194980. doi:10.1001/jamanetworkopen.2019.4980

14. Döpfner M, Breuer D, Schürmann S, Metternich TW, Rademacher C, Lehmkuhl G. Effectiveness of an adaptive multimodal treatment in children with attention-deficit hyperactivity disorder - Global outcome. Eur Child Adolesc Psychiatry. 2004;13(1):i117-i129. doi:10.1007/s00787-004-1011-9

15. Seixas M, Weiss M, Müller U. Systematic review of national and international guidelines on attention-deficit hyperactivity disorder. $J$ Psychopharmacol. 2012;26(6):753-765. doi:10.1177/026988111 1412095
16. Sonuga-Barke EJS, Brandeis D, Cortese S, et al. Nonpharmacological interventions for ADHD: systematic review and meta-analyses of randomized controlled trials of dietary and psychological treatments. Am J Psychiatry. 2013;170(3):275-289. doi:10.1176/ appi.ajp.2012.12070991

17. van Doren J, Arns M, Heinrich H, Vollebregt MA, Strehl U, Loo SK. Sustained effects of neurofeedback in ADHD: a systematic review and meta-analysis. Eur Child Adolesc Psychiatry. 2019;28 (3):293-305. doi:10.1007/s00787-018-1121-4

18. Razoki B. Neurofeedback versus psychostimulants in the treatment of children and adolescents with attention-deficit/hyperactivity disorder: a systematic review. Neuropsychiatr Dis Treat. 2018;14:2905-2913. doi:10.2147/NDT.S178839

19. Duric NS, Assmus J, Gundersen D, Duric Golos A, Elgen IB. Multimodal treatment in children and adolescents with attention-deficit/hyperactivity disorder: a 6-month follow-up. Nord $J$ Psychiatry. 2017;71(5):386-394. doi:10.1080/08039488.2017. 1305446

20. Monastra VJ, Monastra DM, George S. The effects of stimulant therapy, EEG biofeedback, and parenting style on the primary symptoms of attention-deficit/hyperactivity disorder. Appl Psychophysiol Biofeedback. 2002;27(4):231-249. doi:10.1023/ A:1021018700609

21. Enriquez-Geppert S, Smit D, Pimenta MG, Arns M. Neurofeedback as a treatment intervention in ADHD: current evidence and practice. Curr Psychiatry Rep. 2019;21(6). doi:10.1007/ s11920-019-1021-4

22. Arnold LE, Arns M, Barterian J, et al. The neurofeedback collaborative group. double-blind placebo-controlled randomized clinical trial of neurofeedback for attention-deficit/hyperactivity disorder with 13 month follow-up. J Am Acad Child Adolesc Psychiatry. 2020. doi:10.1016/j.jaac.2020.07.906

23. Ros T, Enriquez-Geppert S, Zotev V, et al. Consensus on the reporting and experimental design of clinical and cognitive-behavioural neurofeedback studies (CRED-nf checklist). Brain. 2020;143 (6):1674-1685. doi:10.1093/brain/awaa009

24. Jeunet C, Lotte F, Batail JM, Philip P, Micoulaud Franchi JA. Using recent $\mathrm{BCI}$ literature to deepen our understanding of clinical neurofeedback: a short review. Neuroscience. 2018;378:225-233. doi:10.1016/j.neuroscience.2018.03.013

25. Gevensleben H, Rothenberger A, Moll GH, Heinrich H. Neurofeedback in children with ADHD: validation and challenges. Expert Rev Neurother. 2012;12(4):447-460. doi:10.1586/ern.12.22

26. Sherlin LH, Arns M, Lubar J, et al. Neurofeedback and basic learning theory: implications for research and practice. J Neurother. 2011;15 (4):292-304. doi:10.1080/10874208.2011.623089

27. APA presidential task force on evidence-based practice. Evidencebased practice in psychology. Am Psychol. 2006;61(4):271-285. doi:10.1037/0003-066X.61.4.271

28. Southam-Gerow MA, Prinstein MJ. Evidence Base Updates: the Evolution of the evaluation of psychological treatments for children and adolescents. J Clin Child Adolesc Psychol. 2014;43(1):37-41. doi:10.1080/15374416.2013.855128

29. Tolin DF, Mckay D, Forman EM, Klonsky ED, Thombs BD. Empirically supported treatment: recommendations for a new model. Clin Psychol Sci Pract. 2015;22(4):317-338. doi:10.1111/ cpsp. 12122

30. Arns M, Clark CR, Trullinger M, deBeus R, Mack M, Aniftos M. Neurofeedback and Attention-Deficit/Hyperactivity-Disorder (ADHD) in children: rating the evidence and proposed guidelines. Appl Psychophysiol Biofeedback. 2020;45(2):39-48. doi:10.1007/ s10484-020-09455-2

31. Arns M, Heinrich H, Strehl U. Evaluation of neurofeedback in ADHD: the long and winding road. Biol Psychol. 2014;95:108-115. doi:10.1016/j.biopsycho.2013.11.013 
32. The MTA Cooperative Group. A 14-month randomized clinical trial of treatment strategies for attention-deficit/hyperactivity disorder. Arch Gen Psychiatry. 1999;56(12):1073-1086. doi:10.1001/ archpsyc.56.12.1073

33. Arns M, Vollebregt MA, Palmer D, et al. Electroencephalographic biomarkers as predictors of methylphenidate response in attention-deficit/hyperactivity disorder. Eur Neuropsychopharmacol. 2018;28(8):881-891. doi:10.1016/j.euroneuro.2018.06.002

34. Elliott GR, Blasey C, Rekshan W, et al. Cognitive testing to identify children with ADHD who do and do not respond to methylphenidate. J Atten Disord. 2014;1-10. doi:10.1177/1087054714543924.

35. Swanson JM, Kraemer HC, Hinshaw SP, et al. Clinical relevance of the primary findings of the MTA: success rates based on severity of ADHD and ODD symptoms at the end of treatment. $J$ Am Acad Child Adolesc Psychiatry. 2001;40(2):168-179. doi:10.1097/00004583200102000-00011

36. Steele M, Jensen PS, Quinn DMP. Remission versus response as the goal of therapy in ADHD: a new standard for the field? Clin Ther. 2006;28(11):1892-1908. doi:10.1016/j.clinthera.2006.11.006

37. Arns M, Drinkenburg W, Leon Kenemans J. The effects of QEEG-informed neurofeedback in ADHD: an open-label pilot study. Appl Psychophysiol Biofeedback. 2012;37(3):171-180. doi:10.1007/s10484-012-9191-4

38. Krepel N, Egtberts T, Sack AT, Heinrich H, Ryan M, Arns M. A multicenter effectiveness trial of QEEG-informed neurofeedback in ADHD: replication and treatment prediction. Neuroimage Clin. 2020;28:102399. doi:10.1016/j.nicl.2020.102399

39. Gevensleben H, Holl B, Albrecht B, et al. Neurofeedback training in children with ADHD: 6-month follow-up of a randomised controlled trial. Eur Child Adolesc Psychiatry. 2010;19(9):715-724. doi:10.1007/s00787-010-0109-5

40. Gevensleben H, Holl B, Albrecht B, et al. Is neurofeedback an efficacious treatment for ADHD? A randomised controlled clinical trial. J Child Psychol Psychiatry. 2009;50(7):780-789. doi:10.1111/ j.1469-7610.2008.02033.x

41. Strehl U, Aggensteiner P, Wachtlin D, et al. Neurofeedback of slow cortical potentials in children with Attention-Deficit/Hyperactivity Disorder: a multicenter randomized trial controlling for unspecific effects. Front Hum Neurosci. 2017;11:135. doi:10.3389/ fnhum.2017.00135

42. Aggensteiner P-M, Brandeis D, Millenet S, et al. Slow cortical potentials neurofeedback in children with ADHD: comorbidity, self-regulation and clinical outcomes 6 months after treatment in a multicenter randomized controlled trial. Eur Child Adolesc Psychiatry. 2019;28(8):1087-1095. doi:10.1007/s00787-018-01271-8

43. Kropotov JD, Grin-Yatsenko VA, Ponomarev VA, Chutko LS, Yakovenko EA, Nikishena IS. ERPs correlates of EEG relative beta training in ADHD children. Int J Psychophysiol. 2005;55(1):23-34. doi:10.1016/j.ijpsycho.2004.05.011

44. Steiner NJ, Frenette EC, Rene KM, Brennan RT, Perrin EC. In-school neurofeedback training for ADHD: sustained improvements from a randomized control trial. Pediatrics. 2014;133(3):483-492. doi:10.1542/peds.2013-2059

45. Geladé K, Janssen TWP, Bink M, Van Mourik R, Maras A, Oosterlaan J. Behavioral effects of neurofeedback compared to stimulants and physical activity in attention-deficit/hyperactivity disorder: a randomized controlled trial. J Clin Psychiatry. 2016;77(10): e1270-e1277. doi:10.4088/JCP.15m10149

46. Enriquez-Geppert S, Huster RJ, Scharfenort R, et al. The morphology of midcingulate cortex predicts frontal-midline theta neurofeedback success. Front Hum Neurosci. 2013;7:453. doi:10.3389/fnhum. 2013.00453

47. Sadtler PT, Quick KM, Golub MD, et al. Neural constraints on learning. Nature. 2014;512(7515):423-426. doi:10.1038/ nature 13665
48. Alkoby O, Abu-Rmileh A, Shriki O, Todder D. Can we predict who will respond to neurofeedback? a review of the inefficacy problem and existing predictors for successful EEG neurofeedback learning. Neuroscience. 2018;378:155-164. doi:10.1016/j.neuroscience. 2016.12.050

49. Sagvolden T, Aase H, Zeiner P, Berger D. Altered reinforcement mechanisms in attention-deficit/hyperactivity disorder. Behav Brain Res. 1998;94(1):61-71. doi:10.1016/S0166-4328(97)00 170-8

50. Sonuga-Barke EJS, Taylor E, Sembi S, Smith J. Hyperactivity and delay aversion-I. The effect of delay on choice. J Child Psychol Psychiatry. 1992;33(2):387-398. doi:10.1111/j.1469-7610.1992. tb00874.x

51. Luman M, Oosterlaan J, Hyde C, Van Meel CS, Sergeant JA. Heart rate and reinforcement sensitivity in ADHD. J Child Psychol Psychiatry. 2007;48(9):890-898. doi:10.1111/j.1469-7610.2007. 01769.x

52. Sergeant JA. Modeling attention-deficit/hyperactivity disorder: a critical appraisal of the cognitive-energetic model. Biol Psychiatry. 2005;57(11):1248-1255. doi:10.1016/j. biopsych.2004.09.010

53. Smith AB, Halari R, Giampetro V, Brammer M, Rubia K. Developmental effects of reward on sustained attention networks. Neuroimage. 2011;56(3):1693-1704. doi:10.1016/j.neuroimage. 2011.01.072

54. van Hulst BM, de Zeeuw P, Bos DJ, Rijks Y, Neggers SFW, Durston S. Children with ADHD symptoms show decreased activity in ventral striatum during the anticipation of reward, irrespective of ADHD diagnosis. J Child Psychol Psychiatry. 2017;58(2):206-214. doi:10.1111/jcpp.12643

55. Luman M, Tripp G, Scheres A. Identifying the neurobiology of altered reinforcement sensitivity in ADHD: a review and research agenda. Neurosci Biobehav Rev. 2010;34(5):744-754. doi:10.1016/j. neubiorev.2009.11.021

56. Arnold LE, Lofthouse N, Hersch S, et al. EEG neurofeedback for ADHD: double-blind sham-controlled randomized pilot feasibility trial. $J$ Atten Disord. 2013;17(5):410-419. doi:10.1177/ 1087054712446173

57. Omizo MM, Michael WB, Michael WB, Omizo MM, Michael WB. Biofeedback-induced relaxation training and impulsivity, attention to task, and locus of control among hyperactive boys. J Learn Disabil. 1982;15(7):414-419. doi:10.1177/002221948201500708

58. Braud LW. The effects of frontal EMG biofeedback and progressive relaxation upon hyperactivity and its behavioral concomitants. Biofeedback Self Regul. 1978;3(1):69-89. doi:10.1007/ BF00998565

59. Maurizio S, Liechti MD, Heinrich H, et al. Comparing tomographic EEG neurofeedback and EMG biofeedback in children with attention-deficit/hyperactivity disorder. Biol Psychol. 2014;95:31-44. doi:10.1016/j.biopsycho.2013.10.008

60. Caye A, Swanson JM, Coghill D, Rohde LA. Treatment strategies for ADHD: an evidence-based guide to select optimal treatment. Mol Psychiatry. 2019;24(3):390-408. doi:10.1038/s41380-0180116-3

61. Millichap JG, Yee MM. The diet factor in attention-deficit/hyperactivity disorder. Pediatrics. 2012;129(2):330-337. doi:10.1542/ peds.2011-2199

62. Bijlenga D, Vollebregt MA, Kooij JJS, Arns M. The role of the circadian system in the etiology and pathophysiology of ADHD: time to redefine ADHD? Atten Defic Hyperact Disord. 2019;11 (1):5-19. doi:10.1007/s12402-018-0271-z

63. Arns M, Kenemans JL. Neurofeedback in ADHD and insomnia: vigilance stabilization through sleep spindles and circadian networks. Neurosci Biobehav Rev. 2014;44:183-194. doi:10.1016/j. neubiorev.2012.10.006 
64. Arns M, Feddema I, Kenemans JL. Differential effects of theta/beta and SMR neurofeedback in ADHD on sleep onset latency. Front Hum Neurosci. 2014;8(December):1-10. doi:10.3389/fnhum. 2014.01019
65. Pelham WE, Fabiano GA, Waxmonsky JG, et al. Treatment sequencing for childhood ADHD: a multiple-randomization study of adaptive medication and behavioral interventions. J Clin Child Adolesc Psychol Author. 2016;45(4):396-415. doi:10.1080/15374416.2015.1105138

\section{Publish your work in this journal}

Neuropsychiatric Disease and Treatment is an international, peerreviewed journal of clinical therapeutics and pharmacology focusing on concise rapid reporting of clinical or pre-clinical studies on a range of neuropsychiatric and neurological disorders. This journal is indexed on PubMed Central, the 'PsycINFO' database and CAS, and is the official journal of The International Neuropsychiatric Association (INA). The manuscript management system is completely online and includes a very quick and fair peer-review system, which is all easy to use. Visit http://www.dovepress.com/testimonials.php to read real quotes from published authors. 